\title{
COSTOS Y BENEFICIOS DE LA APLICACIÓN DE ACOLCHADOS EN LA REFORESTACIÓN DE LOS BOSQUES TROPICALES CADUCIFOLIOS
}

\author{
María Guadalupe Barajas-Guzmán y Víctor L. Barradas ${ }^{1}$ \\ Instituto de Ecología, Universidad Nacional Autónoma de México, México, D.F., México \\ ${ }^{1}$ Autor para la correspondencia: vbarrada@ecologia.unam.mx
}

\begin{abstract}
Resumen: Los acolchados incrementan la supervivencia vegetal, favorecen el crecimiento al reforestar las áreas tropicales secas y tornan más eficientes los recursos económicos. En esta investigación se plantearon dos objetivos: (1) determinar a dos años la eficiencia de la reforestación con acolchados y (2) realizar un análisis del costo económico del uso de los acolchados y la generación de plántulas. Para ello, se examinaron los efectos que tendrían los acolchados en la supervivencia y el crecimiento de tres especies nativas en un área degradada de bosque tropical caducifolio. El periodo de estudio fue seco, con la mitad de la precipitación anual media. Se establecieron 48 cuadros con acolchados de paja de alfalfa, hojarasca, polietileno blanco y suelo desnudo. Ipomoea wolcottiana y Caesalpinia eriostachys presentaron el crecimiento y las supervivencias más altas y Lonchocarpus eriocarinalis las más bajas en acolchados de polietileno. La reforestación con el acolchado de polietileno fue menos costosa que con los otros dos.
\end{abstract}

Palabras clave: baja precipitación, Caesalpinia eriostachys, Ipomoea wolcottiana, Lonchocarpus eriocarinalis, restauración ecológica.

\begin{abstract}
Mulches increase the plant survival, promoting growth when reforesting tropical dry areas, and make more efficient economic resources. Two objectives were raised: (1) to determine in two years the efficiency in reforestation and (2) to perform an analysis of the economic cost of using mulches in the reforestation and the generation of the seedlings. Therefore, the effect on survival and growth of three selected native species in a degraded area of a tropical deciduous forest was evaluated. The study period was dry, with half of the average of annual rainfall. Forty eight plots of mulches with alfalfa straw, forest litter, white polyethylene and bare soil, were used. Growth was the highest in the plot of polyethylene mulch than the other treatments. Ipomoea wolcottiana and Caesalpinia eriostachys had the highest growth and survival rates and Lonchocarpus eriocarinalis the lowest in polyethylene mulch. Reforestation with polyethylene mulch was less expensive than the other two.
\end{abstract}

Key words: Caesalpinia eriostachys, ecological restoration, Ipomoea wolcottiana, Lonchocarpus eriocarinalis, low precipitation.

$\mathbf{E}$ n México, el Bosque Tropical Caducifolio (BTC) cubre aproximadamente el $8 \%$ de la superficie del país (Trejo y Dirzo, 2000) y está considerado como un ecosistema amenazado, ya que está sujeto constantemente a perturbaciones muy severas (Murphy y Lugo, 1986). La característica principal de estos bosques es que las plantas pierden sus hojas hacia el inicio de la época seca, debido a la sequedad atmosférica y a la edáfica. La sequedad atmosférica está determinada por una temperatura elevada y una baja humedad relativa del aire (10\% a 20\%) (Salisbury y Ross, 1978), que afecta al organismo vegetal, pues al acrecentarse excesivamente el proceso transpiratorio, perturba la coordinación entre la intensidad de la absorción del agua y su gasto, con lo que la planta se marchita (Baker, 1984). La desecación del suelo es un factor negativo, por lo general se presenta hacia el comienzo de la estación seca, cuando se agotan las reservas de agua. El suelo seco limita la capacidad de las plantas de obtener agua, de manera que los tejidos se deshidratan y el crecimiento se retarda o paraliza del todo (Santos y Ochoa, 1990). Sin embargo, no todo se reduce al agua, ya que debe considerarse que las condiciones y recursos de los cuales dependen las plantas para realizar la fotosíntesis se encuentran en el suelo y en la atmósfera. De manera que las relaciones que se establecen entre suelo-planta-atmósfera, además de las interacciones biológicas, se reflejan en la supervivencia y crecimiento de las plantas (Jones, 1992). 
La temperatura, la luz, el agua disponible, los nutrientes, los recursos maternos y la herbivoría son los principales factores que influyen en el establecimiento y el crecimiento de las plantas (Nobel, 1991). En condiciones naturales, todos estos factores presentan una gran heterogeneidad, y cuando la masa vegetal de cualquier ecosistema terrestre es removida por efecto de la deforestación, se crea un ambiente poco favorable para el establecimiento de las plantas (Ellingson et $a l ., 2000)$. El cambio del uso del suelo se lleva a cabo con la práctica de la roza-tumba-quema. El fuego utilizado, en este procedimiento, cambia el contenido de nutrientes en el suelo, además de reducir la biomasa de las plantas (Kauffman et $a l .$, 2003). En general, el contenido de fósforo y nitrógeno en el suelo se incrementa en el corto plazo; sin embargo, la práctica repetida de la roza y quema incrementa la fijación del fósforo (Ketterings et al., 2002) y disminuye el contenido de nitrógeno (Ellingson et al., 2000), de manera que la fertilidad del suelo declina a mediano plazo.

El movimiento del nitrógeno a través del ecosistema en los BTC es rápido y poco conservativo, lo cual sugiere que no es una limitante en el funcionamiento del bosque. En contraparte, el ciclo del fósforo se caracteriza por una tasa elevada de retraslocación antes de la abscisión de las hojas y, una lenta liberación mediante la descomposición de la materia orgánica. Estas propiedades del ciclo sugieren que el fósforo podría ser limitante en estos bosques (Murphy y Lugo, 1986). De manera que otra de las consideraciones importantes para la reforestación de los BTC es el utilizar alguna técnica que permita retener mayor humedad en el suelo y que además sea fuente de nutrientes.

Una de las técnicas que se utiliza en la agricultura en condiciones muy semejantes a las antes descritas, es la de los acolchados, con la cual se modifica las condiciones físicas del medio y, en general, producen un efecto positivo en el rendimiento de los cultivos (Tilander y Bonzi, 1997; Montague et al., 1998; Lalitha et al., 2010). Los acolchados protegen al suelo de la erosión, reducen el crecimiento de la vegetación (hierbas) no deseada; cuando es orgánico liberan nutrientes al suelo a través de su descomposición, disminuyen la pérdida de humedad del suelo, reducen las fluctuaciones de temperatura e incrementan la apertura estomática (Grantz et al., 1998; Barradas, 2000; Barajas-Guzmán et al., 2006; Barajas-Guzmán y Barradas, 2011). No obstante, la mayoría de estos trabajos se han realizado al corto plazo, o de la estación húmeda a la seca y principalmente en experimentos agrícolas.

Así, el objetivo fundamental de esta investigación fue el de determinar si plantas de especies nativas de un bosque tropical caducifolio podrían llegar a sobrevivir y crecer en zonas perturbadas y con pendientes pronunciadas, al aplicar la técnica de acolchados. Esta técnica podría favorecer cambios en el microambiente atmosférico y edáfico a más largo plazo, por lo que se analiza su efecto en la supervivencia y crecimiento de las especies nativas en un periodo de dos años, además del contenido de agua y nutrientes en el suelo. Conjuntamente se evaluaron los costos económicos que implica un plan de reforestación de este tipo.

\section{Materiales y métodos}

Zona de estudio. El estudio se llevó a cabo en el municipio de San Mateo, en la Costa del Pacífico del estado de Jalisco, México (19 34'07” N, $105^{\circ} 04^{\prime}$ '59" O) en una área dedicada a la ganadería y en donde la vegetación original fue un bosque tropical caducifolio, removida hace aproximadamente 15 años. El paisaje en la zona se encuentra dominado principalmente por Acacia farnesiana (L.) Wild (500 ind ha $^{-1}$ ) con algunos árboles remanentes de Caesalpinia eriosthachys Benth ( 1 ind. ha ${ }^{-1}$ ), y en el estrato herbáceo dominan plantas de la familia Asteraceae.

El clima en la región se identifica con un régimen estacional en la precipitación, presenta una estación lluviosa (junio a octubre) con el $90 \%$ de la precipitación total anual y un patrón irregular en el tiempo (De Ita-Martínez y Barradas, 1986). El promedio anual de precipitación es de 740 mm (García-Oliva et al., 1991). La temperatura media anual es de $24.9{ }^{\circ} \mathrm{C}$, con una variación diurna de $9{ }^{\circ} \mathrm{C}$ en verano y $14{ }^{\circ} \mathrm{C}$ en invierno (De Ita-Martínez y Barradas, 1986). Durante el primer año de estudio la precipitación total anual fue de $391.6 \mathrm{~mm}$ (aproximadamente el 50\% del promedio anual), mientras que el promedio anual de la temperatura mínima fue de $17.2^{\circ} \mathrm{C}$ y la máxima de $28.8^{\circ} \mathrm{C}$.

La vegetación en la región es el bosque tropical caducifolio (Rzedowski, 1978). El bosque es diverso en su composición de especies, con aproximadamente 750 especies agrupadas en 108 familias. Fabaceae es la familia más diversa, contabilizando el 15\% de las especies (Lott et al., 1987). Las especies más comunes son Bursera instabilis Mc Vaugh \& Rzedowski, Caesalpinia eriostachys Benth, Cordia alliodora (Ruiz \& Pav.) Oken, Croton chamelensis Lott, Ipomoea wolcottiana Rose, Jatropha malacophylla Standl., Lonchocarpus eriocarinalis Micheli, y Spondias purpurea L.

El área de estudio se caracteriza por una ladera convexa localizada a $80 \mathrm{~m}$ s.n.m. orientada hacia el sur, y con pendientes que van de 5 a $25^{\circ}$. El suelo (Haplic Ustarents) es poco profundo (en promedio $0.6 \mathrm{~m}$ de profundidad). Las proporciones de arcilla, limo y arena en el suelo a una profundidad de 10 $\mathrm{cm}$ fueron de $25 \pm 3,24 \pm 3$, y $51 \pm 3 \%$ (promedio \pm 1 e.e.), respectivamente (Barajas-Guzmán y Barradas, 2006).

Diseño experimental. Los criterios para la selección de especies fueron: una abundancia de alta a moderada en el bosque maduro; tasa de crecimiento alta, intermedia o lenta; alta producción de semillas y, porcentajes altos de germinación. Las especies que al menos cumplieron con tres de las cuatro características mencionadas fueron: Caesalpinia eriostachys (Fabaceae), Lonchocarpus eriocarinalis (Fabaceae) e Ipomoea wolcottiana (Convolvulaceae). Caesalpinia erios- 
tachys es una especie caducifolia facultativa que pertenece a etapas muy avanzadas en la sucesión y tiene una tasa de crecimiento lenta. Lonchocarpus eriocarinalis es caducifolia y se presenta en zonas perturbadas y dentro del bosque maduro, y es una especie con una tasa de crecimiento intermedia. Ipomoea wolcottiana es una especie caducifolia que se presenta en etapas tempranas de la sucesión y tiene una tasa de crecimiento alta.

Las semillas de estas especies se recolectaron de varios progenitores dentro del BTC en Chamela, Jalisco. Éstas se germinaron en condiciones controladas en un laboratorio del Instituto de Ecología, Universidad Nacional Autónoma de México (UNAM), para obtener información de la latencia y los porcentajes de germinación con condiciones de acuerdo a experiencias previas en las que los porcentajes de germinación fueron mayores al 90\% para las tres especies, y que las semillas de $L$. eriocarinalis y $C$. eriostachys no presentaron latencia, de manera que el único requerimiento para su germinación fue embeberlas durante 24 horas en agua; mientras que las semillas de I. wolcottiana presentaron latencia física y hubo que romperla escarificándolas con $\mathrm{H}_{2} \mathrm{SO}_{4}$ concentrado (Cuadro 1). Posteriormente, se pusieron a germinar 500 semillas de cada especie en bolsas de plástico con suelo proveniente del BTC en un invernadero en la Estación de Biología Chamela, UNAM, en Chamela, Jalisco, para obtener las plántulas para realizar el crecimiento con acolchados.

El experimento se llevó a cabo de julio del 2001 a julio del 2003 , en un área de 4,500 $\mathrm{m}^{2}(90 \times 50 \mathrm{~m})$, orientada principalmente hacia el sur (pendientes de 5 a $25^{\circ}$ ). Se ubicaron tres bloques al azar, cada bloque contenía 16 parcelas de $5 \times 6 \mathrm{~m}$ en las cuales se colocaron tres tipos de acolchado (polietileno blanco, paja de alfalfa (Medicago sativa L.) y hojarasca del bosque tropical caducifolio), utilizando el suelo desnudo como control. La separación entre bloques fue de $2 \mathrm{~m}$, entre parcelas de $1.5 \mathrm{~m}$, y entre cada hilera de parcelas de $4 \mathrm{~m}$ a lo largo de la pendiente. Para prevenir un posible efecto de lixiviación y de escorrentía entre una parcela y otra se construyeron canales perpendiculares a la pendiente ( $85 \mathrm{~m}$ largo, $0.75 \mathrm{~m}$ ancho y $0.60 \mathrm{~m}$ profundo).

Cada parcela con acolchado orgánico se cubrió con 900 $\mathrm{g} \mathrm{m}^{-2}$ de hojarasca o de paja. La hojarasca fue una mezcla de las especies del bosque tropical caducifolio y se recolectó directamente del suelo del bosque en la estación seca, a finales de mayo del 2001. En tanto que, la paja de alfalfa es abundante en la región debido a su utilización como alimento del ganado. El polietileno utilizado fue de color blanco con una vida media de seis meses y se obtuvo en un local comercial.

Entre el 22 y el 25 de julio del 2001 se transplantaron 20 plantas de un año de edad a cada una de las parcelas (0.66 plantas $\mathrm{m}^{-2}$ ), haciendo un total de 240 plantas por especie, con $1.6 \mathrm{~m}$ de distancia entre ellas. En la periferia del cuadro se colocaron 14 plantas y, dentro del mismo seis, éstas últimas fueron las que se tomaron en cuenta para el registro de las medidas de crecimiento y supervivencia de las plantas. Cada parcela contenía a una sola especie. El diseño fue un factorial de 4 ( 3 acolchados y el suelo desnudo) $\times 4$ ( 3 especies y el control sin plantas) con tres réplicas ( 3 bloques) $=48$ parcelas.

Contenido de agua en el suelo, concentraciones de $N$ inorgánico y $P$ lábil, y temperaturas del aire y del suelo. Se recolectaron al azar tres muestras de suelo $(5-10 \mathrm{~cm}$ de profundidad) de cada una de las parcelas a mediados de la época húmeda (septiembre 2001). Estas muestras se combinaron en el campo y se almacenaron a $4{ }^{\circ} \mathrm{C}$ hasta $48 \mathrm{~h}$ antes de procesarlas. Las muestras de suelo se homogeneizaron a mano y se tamizaron $(2 \mathrm{~mm})$ en el laboratorio para la medición de la humedad del suelo (Hs), que se determinó por diferencia de pesos entre el peso húmedo y el seco $\left(105^{\circ} \mathrm{C}\right.$ durante $48 \mathrm{~h}$ ). Con estas muestras también se establecieron las concentraciones de nitrógeno inorgánico $\left(\mathrm{NH}_{4}\right.$ y $\left.\mathrm{NO}_{3}\right)$ y $\mathrm{P}$ lábil. Las concentraciones de $\mathrm{N}$ inorgánico se determinaron por medio de extracción con $\mathrm{KCl} 2 \mathrm{M}$ y las de $\mathrm{P}$ lábil utilizando el método de Murphy y Riley (1962), agitando $0.5 \mathrm{~g}$ de suelo con $30 \mathrm{ml}$ de $\mathrm{NaHCO}_{3} 0.5 \mathrm{M}$ durante $16 \mathrm{~h}$, seguido de un centrifugado a $10,000 \mathrm{rpm}$ a $0{ }^{\circ} \mathrm{C}$ durante 10 min. Las concentraciones de $\mathrm{NH}_{4}, \mathrm{NO}_{3}$ y $\mathrm{P}$ lábil antes de la determinación fueron 9.16, 10.40 y $13.52 \mu \mathrm{g} \mathrm{g}^{-1}$, respectivamente. La temperatura del aire $\left(\mathrm{T}_{\mathrm{A}}\right)$, de la superficie del suelo $\left(\mathrm{Ts}_{0}\right)$ y a la profundidad de $10 \mathrm{~cm}\left(\mathrm{Ts}_{10}\right)$ se midieron entre las 11:00 y 13:00 horas con una sonda de temperatura HMP35C $\left(\mathrm{T}_{\mathrm{A}}\right)$ y HMP107 $\left(\mathrm{Ts}_{0}\right.$ y $\left.\mathrm{Ts}_{10}\right)$ conectadas a una $d a-$ talogger (21X, Campbell Scientific, Logan, Utah).

Cuadro 1. Tiempos de germinación y condiciones a las que se sometieron las semillas de las tres especies nativas en el laboratorio.

\begin{tabular}{|c|c|c|c|c|c|}
\hline Especie & Pretratamiento & $\begin{array}{c}\text { Tiempo de } \\
\text { germinación (día) }\end{array}$ & $\begin{array}{c}\text { Temperatura } \\
\left({ }^{\circ} \mathrm{C}\right)\end{array}$ & $\begin{array}{l}\text { Periodo de } \\
\text { luz-oscuridad }\end{array}$ & $\begin{array}{c}\text { Porcentaje de } \\
\text { germinación (\%) }\end{array}$ \\
\hline C. eriostachys & embebidas en agua $24 \mathrm{~h}$ & $3-4$ & 25 & $12-12$ & 100 \\
\hline L. eriocarinalis & embebidas en agua $24 \mathrm{~h}$ & $7-8$ & 25 & $12-12$ & 95 \\
\hline I. wolcottiana & $\begin{array}{c}\text { escarificación 20-25 min } \\
\text { en } \mathrm{H}_{2} \mathrm{SO}_{4}\end{array}$ & $7-8$ & 25 & $12-12$ & 90 \\
\hline
\end{tabular}


Supervivencia y crecimiento. La supervivencia de las plantas (medida como un porcentaje respecto del número inicial de individuos) se registró después de transcurridos uno y dos años del transplante. El crecimiento se evaluó por medio de las tasas relativas de crecimiento (TRC) de la altura (de la base del tallo hasta el meristemo apical), diámetro (a la altura de la base) y la cobertura foliar (con dos diámetros).

La TRC se calculó de acuerdo a Hunt (1978):

$$
T R C=\left(\ln A_{F}-\ln A_{I}\right) /\left(t_{F}-t_{I}\right)
$$

donde $A_{F}$ y $A_{I}$ son los diámetros, alturas o coberturas finales e iniciales de las plantas; $t_{F}$ es el tiempo final y $t_{I}$ es el tiempo inicial (en días).

Análisis de resultados. Se aplicó un análisis de varianza (ANDEVA) de una vía (tipos de acolchados) a los datos de supervivencia de las tres especies estudiadas (estos últimos fueron transformados al arcoseno de la raíz cuadrada del porcentaje de supervivencia para cumplir con los supuestos de la ANDEVA, Zar, 1999). A los datos de las tasas relativas de crecimiento (TRC): altura, diámetro y cobertura, para cada una de las especies bajo estudio, también se les realizó un ANDEVA de una vía (tipos de acolchados), e igualmente a los datos de microclima. En los tres casos, cuando el análisis mostró diferencias significativas entre acolchados se aplicó la prueba de Tukey (Zar, 1999). En las variables de nutrientes se efectuó un análisis de Kruskall-Wallis, debido a que el número de repeticiones fue bajo y los datos no presentaron una distribución normal (Zar, 1999). Todos los análisis se realizaron con un nivel de confianza del $95 \%$ con un programa computacional (Statistica, 2007).

Indice de costos. Se generó un índice de costos con el que es posible definir si es redituable realizar la inversión económica que representa el uso de los acolchados y la crianza involucrada en la reforestación. Este es un índice de costo (IC) muy sencillo, que está dado por la supervivencia de la especie al menos un año después del trasplante al campo $(\mathrm{S})$, de los costos totales de crianza en el vivero $\left(\mathrm{C}_{\mathrm{TC}}\right)$ y de protección en el campo $\left(\mathrm{C}_{\mathrm{TP}}\right)$ como sigue: $\mathrm{IC}=\mathrm{S} /\left(\mathrm{C}_{\mathrm{TC}}+\right.$ $\mathrm{C}_{\mathrm{TP}}$ ). Desde este punto de vista, la inversión económica se puede recomendar cuando $\mathrm{IC} \geq 0.20$.

\section{Resultados}

Supervivencia. Se presentaron diferencias en la supervivencia de las plantas de Lonchocarpus eriocarinalis entre acolchados $\left(\mathrm{F}_{(3.40)}=14.81 ; P<0.00001\right)$. El porcentaje de supervivencia disminuyó hacia el final de la estación lluviosa en todos los tratamientos (Figura 1). La disminución más drástica se dio en el suelo desnudo, ya que el $60 \%$ de las plantas murieron al finalizar la época de lluvias y después del primer año ninguna planta sobrevivió. Mientras que en los acolchados orgánicos sólo sobrevivió el 46 y $43 \%$ de las plantas (paja de alfalfa y hojarasca del bosque, respectiva-
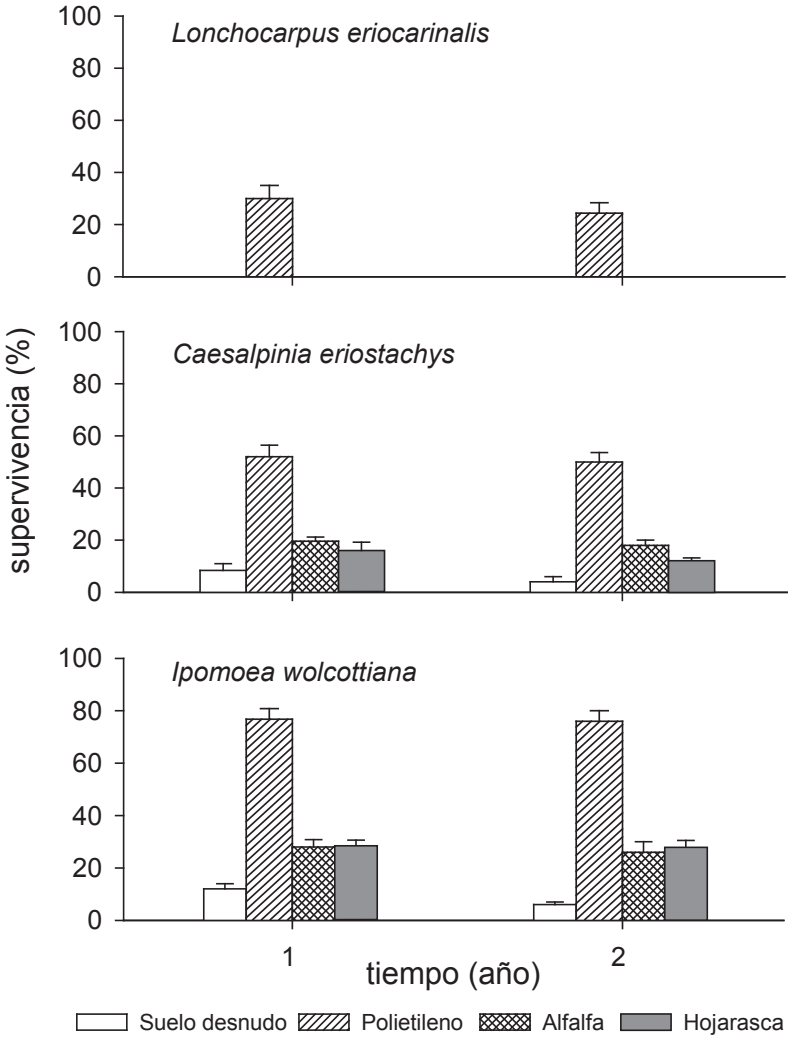

Figura 1. Porcentajes de supervivencia de las plantas de Lonchocarpus eriocarinalis, Caesalpinia eriostachys e Ipomoea wolcottiana después de dos años del trasplante.

mente), al final de las lluvias. Sin embargo, después de un año no hubo sobrevivientes en estos dos acolchados orgánicos. No obstante, en el acolchado de polietileno el $68 \%$ de las plantas de L. eriocarinalis seguían vivas al final de las lluvias, en el primer año el $30 \%$, y después de dos años del trasplante la supervivencia fue del $27 \%$.

En Caesalpinia eriostachys también se presentaron diferencias en la supervivencia de las plantas entre acolchados $\left(\mathrm{F}_{(3,40)}=10.65 ; P<0.00001\right)$. La supervivencia de las plantas de C. eriostachys disminuyó del 22 al 35\% hacia el final de la época de lluvias en todos los acolchados y el suelo desnudo (Figura 1). Sin embargo, después del primer año, los valores más altos de supervivencia (52\%) se registraron en el acolchado con polietileno, mientras que el valor más bajo se registró en el suelo desnudo con un $10 \%$, en tanto que en los acolchados de alfalfa y hojarasca la supervivencia fue de 18 y $15 \%$, respectivamente. Dos años después del transplante, la supervivencia en las parcelas acolchadas con polietileno prácticamente no cambió $(50 \%)$, mientras que los otros tratamientos presentaron una ligera disminución: $15 \%$ en paja de alfalfa, $14 \%$ en hojarasca del bosque y $5 \%$ en el suelo desnudo.

Ipomoea wolcottiana presentó diferencias en la supervivencia entre acolchados $\left(\mathrm{F}_{(3,40)}=57.09 ; P<0.00001\right)$, con 

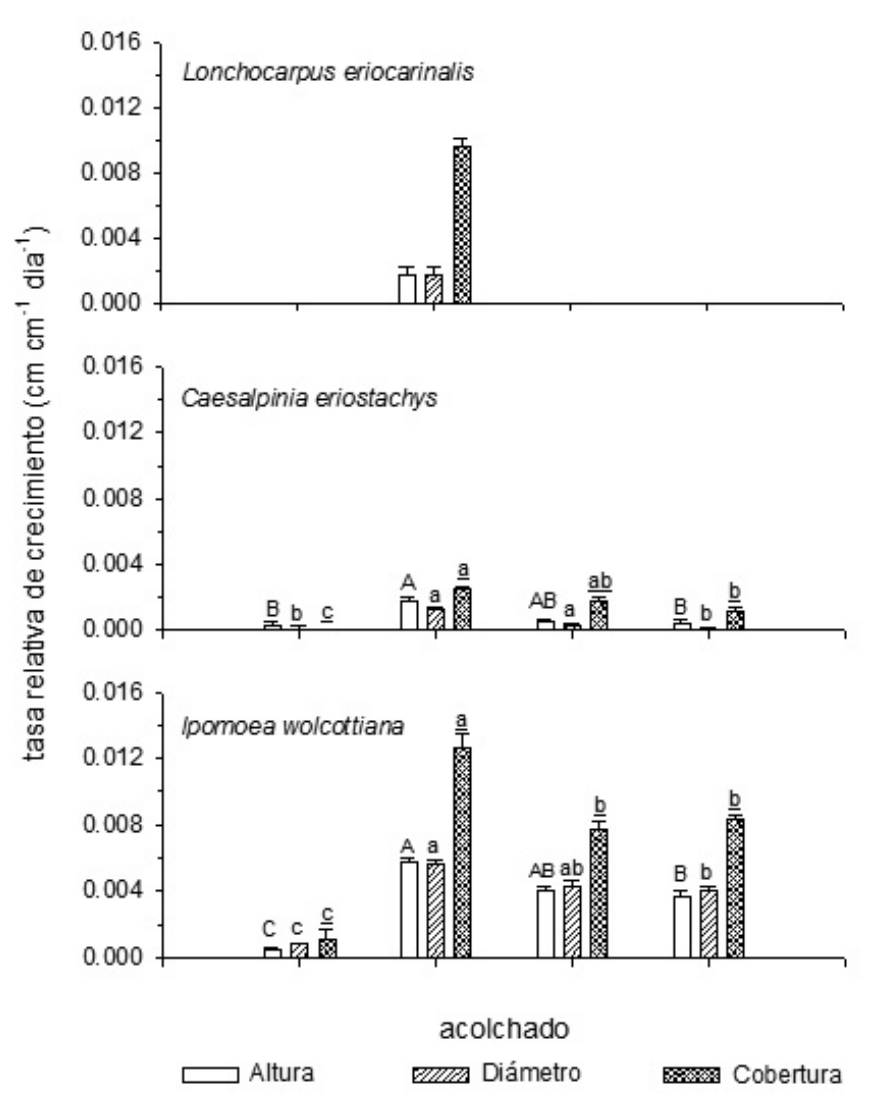

Figura 2. Tasas relativas de crecimiento de la altura, el diámetro a la altura de la base, y la cobertura en las plantas de Lonchocarpus eriocarinalis, Caesalpinia eriostachys e Ipomoea wolcottiana en suelo desnudo (SD) y diferentes acolchados, de polietileno (PO), paja de alfalfa $(\mathrm{AL})$ y hojarasca del bosque $(\mathrm{HO})$ (promedio \pm 1 e.e.). Letras diferentes arriba de las columnas indican diferencias significativas $(P<0.05)$.

porcentajes de supervivencia del 76 y $74 \%$ en el acolchado de polietileno al primer y segundo año después del trasplante, respectivamente. En el suelo desnudo el 5\% de las plantas lograron sobrevivir después de dos años, en tanto que, en los acolchados con paja de alfalfa y con hojarasca del bosque los porcentajes se mantuvieron alrededor del $25 \mathrm{y} \mathrm{el}$ $28 \%$, en el primer y segundo año.
Tasas relativas de crecimiento. Después de un año de crecimiento las plantas de Lonchocarpus eriocarinalis presentaron diferencias significativas en la tasa relativa de crecimiento de las tres variables evaluadas (altura, diámetro y cobertura) entre tratamientos $(P<0.00001$, Figura 2$)$. Las plantas sólo sobrevivieron en el acolchado de polietileno, y por tanto es en este acolchado donde se presentaron valores de crecimiento relativo para los tres parámetros de crecimiento. La altura y el diámetro presentaron una tasa relativa de crecimiento baja pero positiva $\left(0.0016 \mathrm{~cm} \mathrm{~cm}^{-1}\right.$ día $\left.a^{-1}\right)$, mientras que los incrementos más altos se dieron en la cobertura o crecimiento foliar $\left(0.015 \mathrm{~cm} \mathrm{~cm}^{-1}\right.$ día $\left.^{-1}\right)$.

Las plantas de Caesalpinia eriostachys presentaron diferencias significativas en la tasa relativa de crecimiento en la altura $\left(\mathrm{F}_{(3,68)}=9.66 ; P<0.000021\right)$, en el diámetro $\left(\mathrm{F}_{(3,68)}=\right.$ 3.86; $P<0.013)$ y en la cobertura $\left(\mathrm{F}_{(3,68)}=5.76 ; P<0.01\right.$; Figura 2), entre los tres acolchados y el control. Esta especie presentó las tasas de crecimiento más altas en el acolchado de polietileno $\left(0.0015,0.0008,0.0028 \mathrm{~cm} \mathrm{~cm}^{-1} \mathrm{día}^{-1}\right.$, respectivamente), siguiendo en orden decreciente las parcelas con paja de alfalfa, la hojarasca del bosque y los valores más bajos en el suelo desnudo.

Las tasas relativas de crecimiento para las plantas de Ipomoea wolcottiana fueron diferentes $\left(\mathrm{F}_{(3,68)}=20.36 ; 14.20\right.$; 22.0; $P<0.00001$ ) entre tratamientos (Figura 2), las cuales, alcanzaron los valores más altos en el acolchado de polietileno (0.006, 0.0059, $0.013 \mathrm{~cm} \mathrm{~cm}^{-1}$ día $^{-1}$, respectivamente), siendo muy similares los valores alcanzados en los dos acolchados orgánicos (paja de alfalfa y hojarasca), y en el suelo desnudo se dieron tasas bajas para los tres parámetros.

El efecto de los acolchados en el contenido de agua y $\mathrm{nu}$ trientes en el suelo. Los acolchados tuvieron un efecto significativo e importante sobre el microclima y los nutrientes en el suelo (Cuadro 2). Las temperaturas más altas se presentaron en el suelo desnudo, sobre todo en la superficie del suelo hasta con $55^{\circ} \mathrm{C}$, mientras que en el acolchado de polietileno fue $13{ }^{\circ} \mathrm{C}$ menor. En tanto que la humedad del suelo fue semejante en todos los acolchados (0.06-0.07 g $\mathrm{g}^{-1}$ ), a excepción del de polietileno con $0.11 \mathrm{~g} \mathrm{~g}^{-1}$. Por otro lado, los valores más altos de $\mathrm{NO}_{3}$ y $\mathrm{P}$ lábil se registraron en el acolchado de polietileno (11.9 y $15.7 \mu \mathrm{g} \mathrm{g}^{-1}$, respecti-

Cuadro 2. Efecto de los acolchados (sin presencia de plantas) sobre el contenido de agua en el suelo (Hs); la temperatura del suelo en superficie $\left(\mathrm{Ts}_{0}\right)$; y a $10 \mathrm{~cm}$ de profundidad $\left(\mathrm{Ts}_{10}\right)$; la temperatura del aire $\left(\mathrm{T}_{\mathrm{A}}\right)$; y las concentraciones de las formas disponibles de $\mathrm{N}$ y $\mathrm{P}$ para las plantas en la época de lluvias en el año del trasplante.

\begin{tabular}{lccccccc}
\hline Acolchado & $\begin{array}{c}\mathrm{Hs} \\
\left(\mathrm{g} \mathrm{g}^{-1}\right)\end{array}$ & $\mathrm{Ts}_{0}\left({ }^{\circ} \mathrm{C}\right)$ & $\mathrm{Ts}_{10}$ & $\begin{array}{c}\mathrm{T}_{\mathrm{A}} \\
\left({ }^{\circ} \mathrm{C}\right)\end{array}$ & $\begin{array}{c}\mathrm{NH}_{4} \\
\left(\mu \mathrm{g} \mathrm{g}^{-1}\right)\end{array}$ & $\begin{array}{c}\mathrm{NO}_{3} \\
\left(\mu \mathrm{g} \mathrm{g}^{-1}\right)\end{array}$ \\
\hline Suelo desnudo & $0.06^{\mathrm{b}}$ & $55^{\mathrm{a}}$ & $45^{\mathrm{a}}$ & $43^{\mathrm{a}}$ & $2.19^{\mathrm{b}}$ & $8.40^{\mathrm{ab}}$ & $\begin{array}{c}\mathrm{Plábil}_{\left(\mu \mathrm{g}^{-1}\right)} \\
\text { Polietileno }\end{array}$ \\
Paja alfalfa & $0.11^{\mathrm{a}}$ & $42^{\mathrm{c}}$ & $36^{\mathrm{c}}$ & $38^{\mathrm{b}}$ & $2.67^{\mathrm{ab}}$ & $11.90^{\mathrm{a}}$ & $10.1^{\mathrm{b}}$ \\
Hojarasca & $0.07^{\mathrm{ab}}$ & $51^{\mathrm{b}}$ & $37^{\mathrm{b}}$ & $40^{\mathrm{a}}$ & $3.3^{\mathrm{ab}}$ & $4.67^{\mathrm{b}}$ & $11.5^{\mathrm{ab}}$ \\
\hline
\end{tabular}

Letras diferentes en columnas indican diferencias significativas $P<0.05$ 
Cuadro 3. Costo de cada planta establecida en el experimento con acolchados en un bosque tropical caducifolio incluyendo el costo del vivero.

\begin{tabular}{lccc}
\hline Acolchado & $\begin{array}{c}\text { Costo del } \\
\text { material } \\
\text { utilizado } \\
\text { (USD) }\end{array}$ & $\begin{array}{c}\text { Número de plantas } \\
\text { establecidas } \\
\text { (incluyendo las } \\
\text { tres especies) }\end{array}$ & $\begin{array}{c}\text { Costo por } \\
\text { planta } \\
\text { establecida } \\
\text { (USD) }\end{array}$ \\
\hline Suelo desnudo & 0.00 & 10 de 180 & 18.00 \\
Polietileno & 45.00 & 92 de 180 & 2.45 \\
Paja alfalfa & 120.00 & 24 de 180 & 12.50 \\
Hojarasca & 140.00 & 24 de 180 & 13.33 \\
\hline
\end{tabular}

vamente); no siendo así para $\mathrm{NH}_{4}$, cuyo valor más alto se presentó en el acolchado de hojarasca.

Los costos de la reforestación. El costo de cada planta desde su germinación hasta el trasplante al campo fue de aproximadamente 1.00 USD. Mientras que el de los materiales utilizados en el experimento de reforestación con acolchados fue como sigue: el polietileno tuvo un costo de 45.00 , la paja de alfalfa 120.00 y la hojarasca del bosque de 140.00 USD, para toda la superficie acolchada. El cálculo del costo de la hojarasca se realizó basándose en el jornal de un día de trabajo y no por el valor de la misma. El costo menor por planta, sin importar la especie, fue la establecida en el acolchado de polietileno (Cuadro 3).

En un desglose de costos por planta establecida por especie (Cuadro 4) se comprobó que el mejor tratamiento fue el acolchado de polietileno, con el costo más bajo en com-

Cuadro 4. Costo por planta sobreviviente de cada una de las especies utilizadas en la reforestación con acolchados en un bosque tropical caducifolio incluyendo el costo de vivero. El símbolo * significa una pérdida económica total debido a que en su caso ninguna planta sobrevivió.

\begin{tabular}{llccc}
\hline Acolchado & Especie & $\begin{array}{c}\text { Costo del } \\
\text { material } \\
\text { utilizado } \\
\text { por especie } \\
\text { (USD) }\end{array}$ & $\begin{array}{c}\text { Número de } \\
\text { plantas } \\
\text { sobrevivientes } \\
\text { por especie }\end{array}$ & $\begin{array}{c}\text { Costo de } \\
\text { cada planta } \\
\text { sobreviviente } \\
\text { (USD) }\end{array}$ \\
\hline Suelo & C. eriostachys & 0 & 5 de 60 & 12.00 \\
desnudo & L. eriocarinalis & 0 & 0 de 60 & $60.00^{*}$ \\
& I. wolcottiana & 0 & 5 de 60 & 12.00 \\
Polietileno & C. eriostachys & 15.00 & 30 de 60 & 2.50 \\
& L. eriocarinalis & 15.00 & 17 de 60 & 4.41 \\
& I. wolcottiana & 15.00 & 45 de 60 & 1.66 \\
Paja alfalfa & C. eriostachys & 40.00 & 9 de 60 & 11.10 \\
& L. eriocarinalis & 40.00 & 0 de 60 & $100.00^{*}$ \\
& I. wolcottiana & 40.00 & 15 de 60 & 6.67 \\
& C. eriostachys & 46.70 & 8 de 60 & 13.34 \\
& L. eriocarinalis & 46.70 & 0 de 60 & $106.70^{*}$ \\
& I. wolcottiana & 46.70 & 16 de 60 & 6.67 \\
\hline
\end{tabular}

paración con los demás tratamientos. En cuanto a especie, Ipomoea wolcottiana fue la más económica y más aún, con el acolchado de polietileno.

Se encontró que los valores de IC fueron, en el caso del acolchado de polietileno, 0.50 para Ipomoea wolcottiana, 0.22 para Lonchocarpus eriocarinalis y 0.40 para Caesalpinia eriostachys, mientras que en el suelo desnudo los valores fueron de $0.083,0.0$ y 0.083 , respectivamente. Los valores para los otros acolchados (alfalfa y hojarasca) fueron menores de 0.20 .

\section{Discusión}

Es evidente que el acolchado de polietileno redujo de manera importante la temperatura del aire y del suelo, así como la evaporación, ya que fue en este acolchado donde se registró el valor más alto de humedad en el suelo. También es interesante observar que bajo este acolchado se alcanzaron los valores más altos de nitratos y fósforo lábil, muy probablemente debido a una mayor actividad de microorganismos producida por las relativamente temperaturas bajas del suelo y mayor humedad. No obstante, el valor más alto de $\mathrm{NH}_{4}$ se presentó en el acolchado de hojarasca y relativamente más alto en el acolchado de paja de alfalfa aunque no hubo diferencias con el acolchado de polietileno, debido probablemente a una mayor incorporación al suelo desde los acolchados.

Lonchocarpus eriocarinalis fue la especie más sensible a las condiciones de estrés que prevalecieron durante el trasplante en las parcelas experimentales, ya que las plantas de esta especie perdieron todas sus hojas y parte del tallo dos días después del trasplante mientras se acolchaban las parcelas. En general, su desarrollo fue bajo a pesar de ser una especie que presenta nódulos bacterianos y que puede deshacerse de los órganos que la hacen perder agua y sobrevivir.

Las plantas de Caesalpinia eriostachys fueron exitosas en su establecimiento, pues en todos los acolchados y en el suelo desnudo lograron sobrevivir, aunque los valores más altos se dieron en el acolchado de polietileno. Sin embargo, debido a su tasa lenta de crecimiento es poco recomendable para la recuperación de zonas degradadas, al menos durante la primera etapa. Los valores bajos de supervivencia y de crecimiento tanto de Caesalpinia eriostachys como de Ipomoea wolcottiana en el suelo desnudo, demuestran que los factores limitantes más importantes, en este tipo de bosque, son la baja disponibilidad de agua y las temperaturas altas; aunque los valores de $\mathrm{NH}_{4}\left(2.19\right.$ - $\left.5.8 \mu \mathrm{g} \mathrm{g}^{-1}\right)$ se presentaron muy por debajo del umbral $\left(25-50 \mu \mathrm{g} \mathrm{g}^{-1}\right)$ estipulado por Binkley y Vitousek (1989) para un buen desempeño vegetativo. Aun así, Caesalpinia eriostachys fue la más "perenne" de las tres especies estudiadas, ya que mantuvo su follaje por más tiempo. 
Ipomoea wolcottiana fue la especie que mejor respondió a los tratamientos de acolchado, probablemente debido a su tasa de crecimiento alta. Aunque su desarrollo fue bueno en los acolchados orgánicos, su mejor desempeño se dio, nuevamente, en el acolchado de polietileno. Este resultado indica que es importante el introducir especies de rápido crecimiento como I. wolcottiana al inicio de la recuperación de las áreas degradadas. Otra característica que exhibe esta especie, es la presencia de raíces tuberosas que le permiten, probablemente, almacenar agua, lo cual es importante, pues los sistemas radicales tienen un papel preponderante en la dotación de agua y nutrientes a las plantas.

Los resultados de esta investigación son semejantes a los reportados por Lalitha et al. (2010) en cultivos y, a los encontrados por Blanco-García y Lindig-Cisneros (2005) con acolchados de corteza de pino que incrementaron la supervivencia de Pinus pseoudostrobus en áreas cubiertas de tefra volcánica en la Mesa de Cuitzeo. Sin embargo, en otra región de Michoacán (Nuevo San Juan Parangaricutiro) este mismo tipo de acolchado no presentó ningún efecto en la supervivencia de P. pseudostrobus y Abies religiosa (Blanco-García et al., 2011).

Por otro lado, y en este caso, resulta sumamente importante notar que hubo cambios mínimos en el porcentaje de supervivencia de un año al otro, de manera que este hecho nos permite indicar que es suficiente acolchar en una sola temporada de crecimiento (época de lluvias), para asegurar el incremento significativo en la supervivencia a un plazo mayor de un año.

Sin embargo, para efectos de una estrategia de reforestación es necesario saber si es rentable reforestar con plantas combinadas con acolchados. Resulta evidente que lo más barato es reforestar utilizando el acolchado de polietileno, y que probablemente ningún programa de reforestación estaría dispuesto a invertir más de $\$ 5.00$ USD por planta, ya que es un precio alto para un sólo individuo. Tampoco resultaría conveniente plantar sin acolchar, ya que la supervivencia es baja (5.5\%). Aunque si se desglosan los costos por especie el panorama cambia (Cuadro 4), porque es más barato el costo por planta si se utiliza el polietileno en especies con rápido crecimiento. En contraparte, es de alto costo reforestar con paja u hojarasca, a pesar de que la especie sea de rápido crecimiento; sin embargo, es viable utilizar acolchados.

Los valores de IC desarrollados en este trabajo, demuestran que es redituable invertir en la reforestación utilizando acolchados de polietileno sin depender de la especie, aunque si se utilizan especies de rápido crecimiento la inversión es más baja. Sin embargo, la inversión no es redituable si se utilizan los otros acolchados (alfalfa y hojarasca), no importando la especie.

Si se aplica este índice a los datos presentados en la investigación realizada por Holl y Quiroz-Nietzen (1999) en Costa Rica, se encuentra que la inversión en la crianza en vi- vero y en la protección de Ocotea glaucosericea, O. whitei, Sideroxylin portoricensi y Vochysia alleni contra herbivoría por conejos, se recomienda para las cuatro especies. Aunque los costos de crianza en Costa Rica fueron hasta 5.7 veces más bajos que los de Jalisco pero los costos de la protección fueron un poco más alto ( 0.2 veces).

La utilización de un índice de costo como el de este trabajo o como el presentado por Martínez-Ramos y GarcíaOrth (2007) sólo puede dar un indicio para recomendar o no la reforestación con especies nativas. Un índice formal sería aquel que involucre los costos y los beneficios de la reforestación, desafortunadamente el valor económico del beneficio no ha sido posible todavía establecerlo.

Este trabajo demuestra, que una buena estrategia de reforestación para los BTC, es la utilización de un acolchado de polietileno, el cual tendría que ser blanco, para disminuir la radiación neta y por ende, disminuir la temperatura del suelo y del aire. El uso de esta técnica repercutiría de manera favorable en la humedad y la concentración de nutrientes en el suelo, pues no es suficiente con reducir sólo el déficit hídrico, ya que la temperatura juega un papel determinante en la supervivencia y crecimiento de las plantas (Barajas-Guzmán y Barradas, 2011). Además de que los acolchados amplían la disponibilidad de nutrientes. Es necesario recalcar que la reforestación asistida, tan costosa; como la natural (sucesión), tan valiosa, no tendrán el éxito deseado mientras subsistan las causas de la destrucción de los bosques, como son los desmontes para la agricultura, el pastoreo extensivo, las talas clandestinas, los incendios forestales y sobre todo el crecimiento acelerado de la población.

\section{Agradecimientos}

Agradecemos a L. Hernández, G. Prado y D. Juárez por asistencia técnica. También agradecemos a dos revisores anónimos sus acertados comentarios y sugerencias. Esta investigación se realizó con el apoyo económico otorgado por DGAPA-PAPIIT, UNAM (IN204599) y CONACyT (MGBG).

\section{Literatura citada}

Baker D.A.1984. Water relations. En: Wilkins M.B. Ed. Advanced Plant Physiology, pp. 297-318, Pitman Publishing, Londres.

Barajas-Guzmán M.G. y Barradas V.L. 2011. Microclimate and sapling survival under organic and polyethylene mulch in a tropical dry deciduous forest. Boletín de la Sociedad Botánica de México 88:27-34.

Barajas-Guzmán M.G., Campo J. y Barradas V.L. 2006. Soil water, nutrient availability and sapling survival under organic and polyethylene mulch in a seasonally dry tropical forest. Plant and Soil 287:347-357.

Barradas V.L. 2000. Modificación del microclima con énfasis en la conservación y la restauración ecológica. Boletín de la Sociedad Botánica de México 65:83-88. 
Binkley D. y Vitousek P. 1989. Soil nutrient availability. En: Pearcy R.W., Ehleringer J.R, Mooney H.A. y Rundel P.W. Eds. Plant Physiological Ecology: Field Methods and Instrumentation, pp 75-96, Chapman and Hall, Londres.

Blanco-García A. y Lindig-Cisneros R. 2005. Incorporating restoration in sustainable forestry management: using pine-bark mulch to improve native species establishment on tephra deposits. Restoration Ecology 13:703-709.

Blanco-García A., Sáenz-Romero C., Martorell C., Alvarado-Sosa P. y Lindig-Cisneros R. 2011. Nurse-plant and mulching effects on three conifer species in a Mexican temperate forest. Ecological Engineering 37:994-998.

De Ita-Martínez C. y Barradas V.L. 1986. El clima y los patrones de producción agrícola en una selva baja caducifolia de la costa de Jalisco, México. Biótica 11:237-245.

Ellingson L.J., Kauffman J.B, Cummings D.L., Sanford R.L. y Jaramillo V.J. 2000. Soil N dynamics associated with deforestation, biomass burning, and pasture conversion in a Mexican tropical dry forest. Forest Ecology and Management 137:41-51.

García-Oliva F., Ezcurra E. y Galicia L.1991. Pattern of rainfall distribution in the Central Pacific Coast of México. Geografiska Annalizer. Series A, Physical Geography 73:179-186.

Grantz D.A., Vaughn D.L., Farber R.J., Kim B., Ashbaugh L., VanCuren T., Campbell R., Bainbridge D. y Zink T. 1998. Transplanting native plants to revegetate abandoned farmland in the western Mojave desert. Journal of Environmental Quality 27:960-967.

Holl K.D. y Quiroz-Nietzen E. 1999. The effect of rabbit herbivory on reforestation of abandoned pasture in southern Costa Rica. Biological Conservation 87:391-395.

Hunt R.1978. Plant Growth Analysis. Edward Arnold, Londres.

Jones H.G.1992. Plants and Microclimate. A Quantitative Approach to Environmental Plant Physiology. 2a ed. Cambridge University Press, Cambridge.

Kauffman J.B., Steele M.D., Cummings D.L. y Jaramillo V.J. 2003. Biomass dynamics associated with deforestation, fire, and conversion to cattle pasture in a Mexican tropical dry forest. Forest Ecology and Management 176:1-12.
Ketterings Q.M., van Noordwijk M. y Bigham J.M. 2002. Soil phosphorus availability after slash-and-burn fires of different intensities in rubber agroforests in Sumatra, Indonesia. Agriculture, Ecosystems \& Environment 92:37- 48.

Lalitha M., Thilagham V.K., Balakrishnan N. y Mansour M. 2010. Effect of plastic mulch on soil properties and crop growth - A review. Agricultural Reviews 31:145-149.

Lott E.J., Bullock S.H. y Solís-Magallanes J.A. 1987. Floristic diversity and structure of upland and arroyo forest of coastal Jalisco. Biotropica 19:228-235.

Martínez-Ramos M. y García-Orth X. 2007. Sucesión ecológica y restauración de las selvas húmedas. Boletín de la Sociedad Botánica de México 80 (Supl.):69-84.

Montague T., Kjelgren R. y Rupp L. 1998. Surface energy balance affects gas exchange of three shrub species. Journal of Arboriculture 24:254-262.

Murphy P.G. y Lugo A.E. 1986. Ecology of tropical dry forest. Annual Review of Ecology and Systematics 17:67-88.

Murphy J. y Riley J.P. 1962. A modified single solution method for the determination of phosphate in natural waters. Analytica Chimica Acta 27:3-36

Nobel P.S. 1991. Physicochemical and Environmental Plant Physiology. Academic Press, San Diego.

Rzedowski J. 1978. Vegetación de México. Limusa, México, D.F.

Salisbury F.B. y Ross C.W. 1978. Plant Physiology. $2^{\mathrm{a}}$ ed., Wadsworth Publishing Company, Belmont.

Santos D.M.S. y Ochoa A.N.1990. Adaptación de las plantas al déficit hídrico. Ciencia 41:333-344.

Statistica. 2007. Statistica versión 8. Statsoft, Tulsa.

Trejo I. y Dirzo R. 2000. Deforestation of seasonally dry tropical forest: a national and local analysis in México. Biological Conservation 94:133-142

Tilander Y. y Bonzi M.1997. Water and nutrient conservation through the use of agroforestry mulches, and sorghum yield response. Plant and Soil 197:219-232.

Zar J.H. 1999. Biostatistical Analysis. Prentice Hall, Upper Saddle River.

Recibido: 25 de mayo de 2012.

Aceptado: 18 de noviembre de 2012. 